\title{
Chapter 14 \\ Emerging Trends and Future Perspective of Novel Cardiac SPECT Technology
}

\author{
Masao Miyagawa, Yoshiko Nishiyama, Hayato Ishimura, \\ Rami Tashiro, Kana Ide, and Teruhito Mochizuki
}

\begin{abstract}
In response to concerns about overuse and increasing radiation exposure of myocardial perfusion imaging, nuclear medicine societies have declared statements aimed at lowering its radiation dose and costs. Simultaneously, two vendors have launched novel SPECT scanners with solid-state semiconductor detectors. Discovery NM 530c and D-SPECT utilize the same cadmium zinc telluride (CZT) detectors with a different combination of high-sensitivity multi-pinhole or parallelhole collimator which focuses on the heart. The physical performance of those is dramatically higher than that of conventional Anger cameras; however, 2 CZT cameras are inherently different.

Although ${ }^{99 \mathrm{~m}}$ Tc-labeled myocardial perfusion tracers might not be ideal agents, estimation of absolute myocardial blood flow or myocardial flow reserve (MFR) using dynamic CZT SPECT is a challenging subject and attracts a great deal of interest in the field. Thus, novel software which allows automatic calculation of MFR index with dynamic CZT SPECT is currently under development and validated in our institution. This technology will hold promise if the several issues can be solved through future studies.
\end{abstract}

Keywords Cadmium zinc telluride - Cardiac SPECT - Coronary flow reserve • Effective dose $\bullet$ Dynamic imaging

\subsection{Introduction}

The overall utilization for stress myocardial perfusion imaging (MPI) reached its height in 2006 with over 10 million studies and Medicare payment over 1 billion dollars [1]. The National Council on Radiation Protection and Measurements

M. Miyagawa, MD, Ph.D. (西) • Y. Nishiyama, MD, Ph.D. • H. Ishimura, RT •

R. Tashiro, MD, Ph.D. • K. Ide, MD, Ph.D. • T. Mochizuki, MD, Ph.D.

Department of Radiology, Ehime University Graduate School of Medicine, Shitsukawa,

Toon-city, Ehime 791-0295, Japan

e-mail: miyagawa@m.ehime-u.ac.jp

(C) The Author(s) 2016 
reported that since the early 1980s, a six-fold increase in radiation exposure to the US population from medical procedures [2] emphasizes that more than $10 \%$ of the entire radiation burden was related to MPI [3]. In response to these concerns, professional societies have declared statements aimed at lowering its radiation dose and costs $[4,5]$.

Simultaneously, vendors have developed dedicated cardiac SPECT scanners with solid-state semiconductor detectors in order to do something about radiation dose and long imaging time. Two vendors have introduced novel scanners: Discovery NM 530c, (D530c); GE Healthcare and D-SPECT; and Spectrum Dynamics utilizing the same cadmium zinc telluride (CZT) detectors, with a different combination of high-sensitivity multi-pinhole or parallel-hole collimators which focuses on the myocardium $[6,7]$. More than 300 of such cardiac CZT SPECT scanners are currently available in the world, and the number is increasing by more than 100 per year.

Firstly, we focus on the latest advances in MPI procedures which ought to be able to maximize the value of SPECT cameras with CZT detectors. Secondly, we try to develop a novel software for calculating the myocardial flow reserve (MFR) index using these cameras and to validate its utility for screening patients with multi-vessel coronary artery disease (CAD).

\subsection{Materials and Methods}

Initially, we conducted a comparative study using ${ }^{99 \mathrm{~m}} \mathrm{Tc}$ line-source phantoms with and without photon scattering caused by water in the cylinder [8]. D530c had a more than two-fold spatial resolution than did the conventional Anger camera with a dual detector (Infinia; GE Healthcare). We also found that a D530c has better energy resolution compared to Infinia, which is as narrow as $5 \%$. Therefore, energy window width could be narrowed enough to be feasible for performing dual radioisotope simultaneous SPECT with ${ }^{99 \mathrm{~m}}$ Tc-tetrofosmin and ${ }^{123}$ I-BMIPP [9].

MPI protocol for routine clinical practice of our institution at present is summarized in Fig. 14.1. We adopt a stress-first one-day protocol with ${ }^{99 \mathrm{~m}}$ Tc-perfusion tracers. CZT camera has a 5-times higher sensitivity; therefore, patients are given an injection of only $3 \mathrm{MBq} / \mathrm{kg}$ body weight $(4-5 \mathrm{mCi})$ of ${ }^{99 \mathrm{~m}} \mathrm{Tc}$-perfusion tracers at peak stress. Supine and prone imaging with $5 \mathrm{~min}$ each is routinely performed [10]. Same-day rest imaging was performed after $9 \mathrm{MBq} / \mathrm{kg}$ of tracer injection. If we apply a strategy of stress-only protocol to our routine clinical practice, we will get further reduction in the effective radiation dose of patients and higher laboratory throughput.

Dynamic MPI starting with the bolus administration of ${ }^{99 \mathrm{~m}}$ Tc-perfusion tracers as described above was also performed during adenosine stress and at rest using the CZT camera. We intravenously injected saline followed by radionuclide, using an 


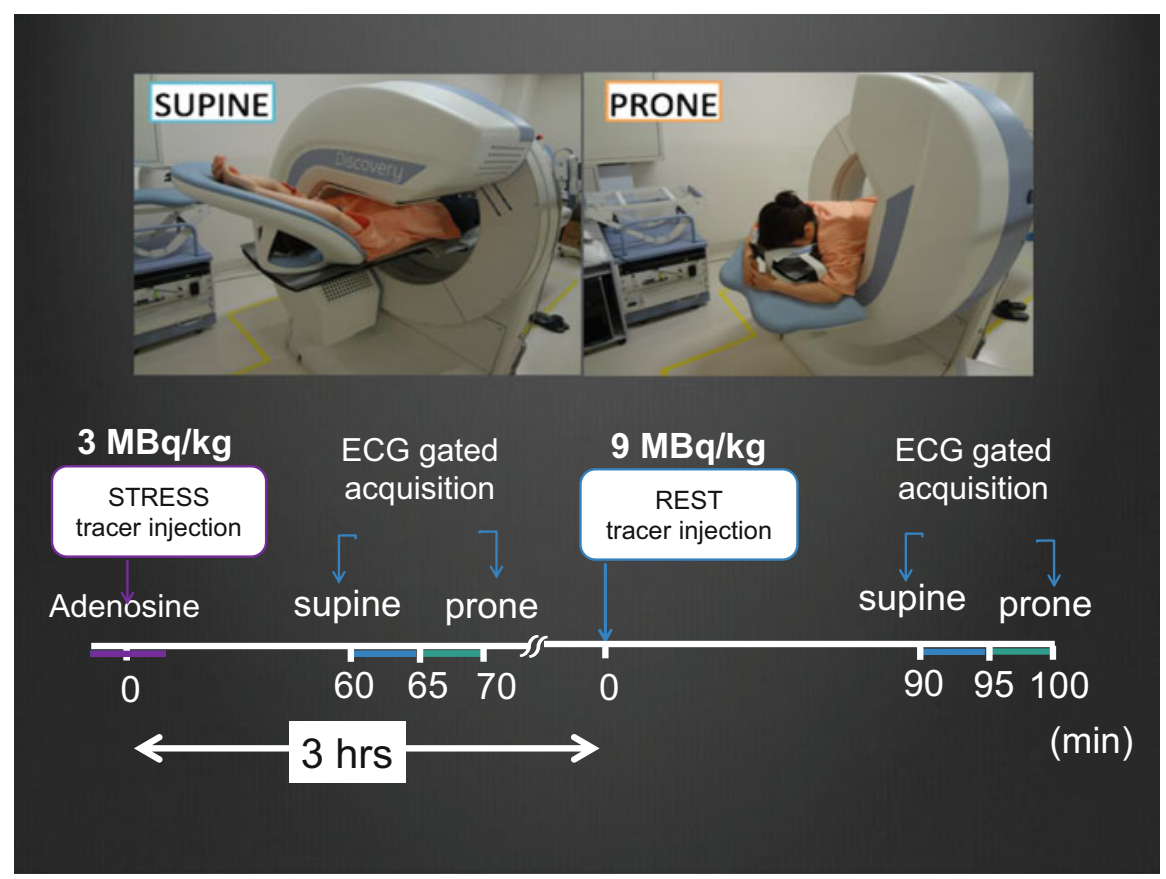

Fig. 14.1 We adopt a stress/rest one-day protocol with Tc-99m perfusion radiotracers. Supine and prone positioning are routinely performed. The total effective dose ranged 3.4-6.7 mSv. Stressonly MPI can be performed with the effective dose averaging $1 \mathrm{mSv}$

automatic injector at a constant rate of 1 milliliter per second for $30 \mathrm{~s}$ (Fig. 14.2). Before dynamic imaging, we carefully detected the border of the heart by chest percussion or test injection of $0.5 \mathrm{mCi}$ of tracers in order to adjust the position of the heart appropriately in the quality field of view [11]. The interval between stress and rest imaging was $3 \mathrm{~h}$ and a 30-s pre-scan count was subtracted from the dynamic data at rest. 10-min dynamic SPECT data with list mode acquisition were reconstructed using a maximum likelihood expectation maximization (MLEM) algorithm with 40-50 iterations. We generated 200 3-D volumes integrating 3-s time frames in the course of $600 \mathrm{~s}$. Routine MPI were also acquired thereafter.

The software allows the automatic edge detection of volume of interest for the blood pool in the left ventricle and the myocardium. Global time-activity curves were fitted to a one-tissue two-compartment kinetic model (2-com), a Patlak plot analysis (PPA), and a dose uptake ratio of MPI (DUR) with input function. K1 and K2 were calculated for the stress and rest images. MFR index was calculated as follows: $\mathrm{MFR}$ index $=\mathrm{K} 1 \mathrm{stress} / \mathrm{K} 1$ at rest.

The validation study included 64 consecutive pts who underwent CZT SPECT and invasive coronary angiography within 2 weeks ( 35 males, $67 \pm 10$ years old). 15 pts had single-vessel CAD, and 22 pts had multi-vessel CAD (10 had two-vessel and 12 had three-vessel CAD) and 27 pts with no significant coronary stenosis less than $70 \%$. 

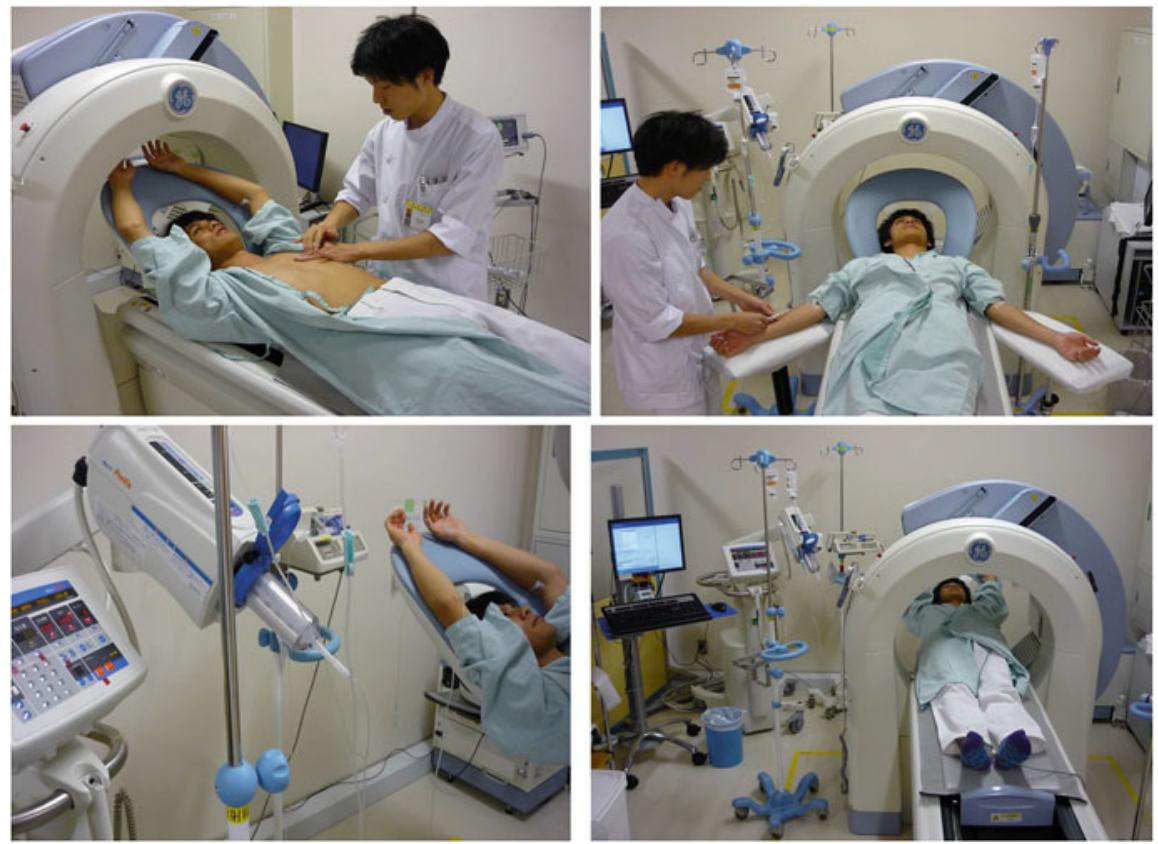

Fig. 14.2 For bolus administration of tracer, we intravenously injected saline, using an automatic injector at a constant rate of 1 milliliter per second for $30 \mathrm{~s}$. We started the dynamic data acquisition just after the bolus injection

\subsection{Results and Discussion}

\subsubsection{Reduction in Injection Dose of Radiopharmaceuticals}

The introduction of CZT cameras has opened the possibility of reducing radiation dose of SPECT MPI. Oddstig et al. [12] reported that they performed a 1-day ${ }^{99 \mathrm{~m}} \mathrm{Tc}-$ tetrofosmin stress-rest protocol using D530c in 150 patients who were divided into three subgroups (50 patients in each group) with 4,3 , and $2.5 \mathrm{MBq} / \mathrm{kg}$ body weight of administered activity in the stress MPI, respectively. The total effective dose (stress and rest) decreased from $9.3 \mathrm{mSv}$ in the $4 \mathrm{MBq} / \mathrm{kg}$ group to $5.8 \mathrm{mSv}$ in the $2.5 \mathrm{MBq} / \mathrm{kg}$ group. The image acquisition times for $2.5 \mathrm{MBq} / \mathrm{kg}$ were 8 and $5 \mathrm{~min}$ (stress and rest, respectively) compared to $15 \mathrm{~min}$ for each when using conventional SPECT. The average image quality for the stress and the rest showed no statistically significant difference among the 4,3 , and $2.5 \mathrm{MBq} / \mathrm{kg}$ groups.

Starting the MPS protocol with examination at stress and analyzing the stress images before deciding of the need for rest examination (i.e., to say "stress-only protocol") reduce the effective dose $[13,14]$. The effective dose was no more than $1.4 \mathrm{mSv}$ for a patient receiving $2.5 \mathrm{MBq} / \mathrm{kg}$, who underwent the stress-only 
protocol. The total effective radiation dose ranged 3 to $6 \mathrm{mSv}$ with the stress-rest protocol in our institution. If applied, stress-only protocol would be performed with the effective dose averaging $1 \mathrm{mSv}$. Novel CZT technology can considerably decrease the effective dose for MPI with preserved high image quality.

Moreover, the high sensitivity of D530c allows for a shorter acquisition time; actually, $5 \mathrm{~min}$ is sufficient for QGS in the clinical setting. Although CZT detectors are higher in cost, they have been shown to provide an eight- to ten-fold increase in sensitivity, coupled with a two-fold improvement in spatial resolution, and higher energy resolution enabling a significant reduction in imaging time and dose of isotopes and a dual radionuclide simultaneous SPECT with ${ }^{99 \mathrm{~m}} \mathrm{Tc}$ and ${ }^{123} \mathrm{I}[6,7]$.

Imbert et al. also conducted analyses of phantom and human SPECT images, comparing the D530c and D-SPECT CZT cameras with Anger cameras, and reported that the physical performance of CZT cameras is dramatically higher than that of Anger cameras; however, 2 CZT cameras are inherently different. Spatial resolution and contrast-to-noise ratio are better with the Discovery NM $530 \mathrm{c}$, whereas detection sensitivity is markedly higher with the D-SPECT [15].

\subsubsection{Attempts to Estimate Coronary Flow Reserve Using CZT SPECT}

At present, coronary flow reserve (CFR) has been mostly replaced by FFR primarily due to its technical simplicity in the catheterization lab. In contrast, in the noninvasive field, PET-derived CFR is an emerging index used for improving both the diagnosis and risk stratification in patients with suspected CAD. And with the increased use, reliable evidences have been suggested that CFR is a powerful independent predictor of cardiac events and mortality [16-19].

In parallel to the work on these PET studies, some studies have continuously reported that using first-pass planar scintigraphy in humans [20-24] or dynamic SPECT in animals [25] provides evidences that the estimates of CFR can be also derived from conventional SPECT MPI. Although fair agreements have been noted between CFR estimated by SPECT and PET or intracoronary Doppler flow studies, they also highlighted the limitations of conventional gamma camera for the dynamic data collection during rapidly changing radiotracer concentrations [26].

The CZT cameras provide higher temporal and spatial resolution. Dynamic SPECT imaging during the first pass of a tracer was attained with the use of these cameras. From the list data, time-activity curves (TAC) would be generated for the left ventricular cavity (input function) and for myocardial tissue (output function) during stress and rest. Ben-Haim et al. [27] firstly reported the feasibility of dynamic ${ }^{99 \mathrm{~m}}$ Tc-MIBI SPECT and quantitation of global and regional CFRs using the D-SPECT. They calculated CFR index as the ratio of the stress and rest K1 values. Global CFR index was higher in patients with normal MPI than in patients 


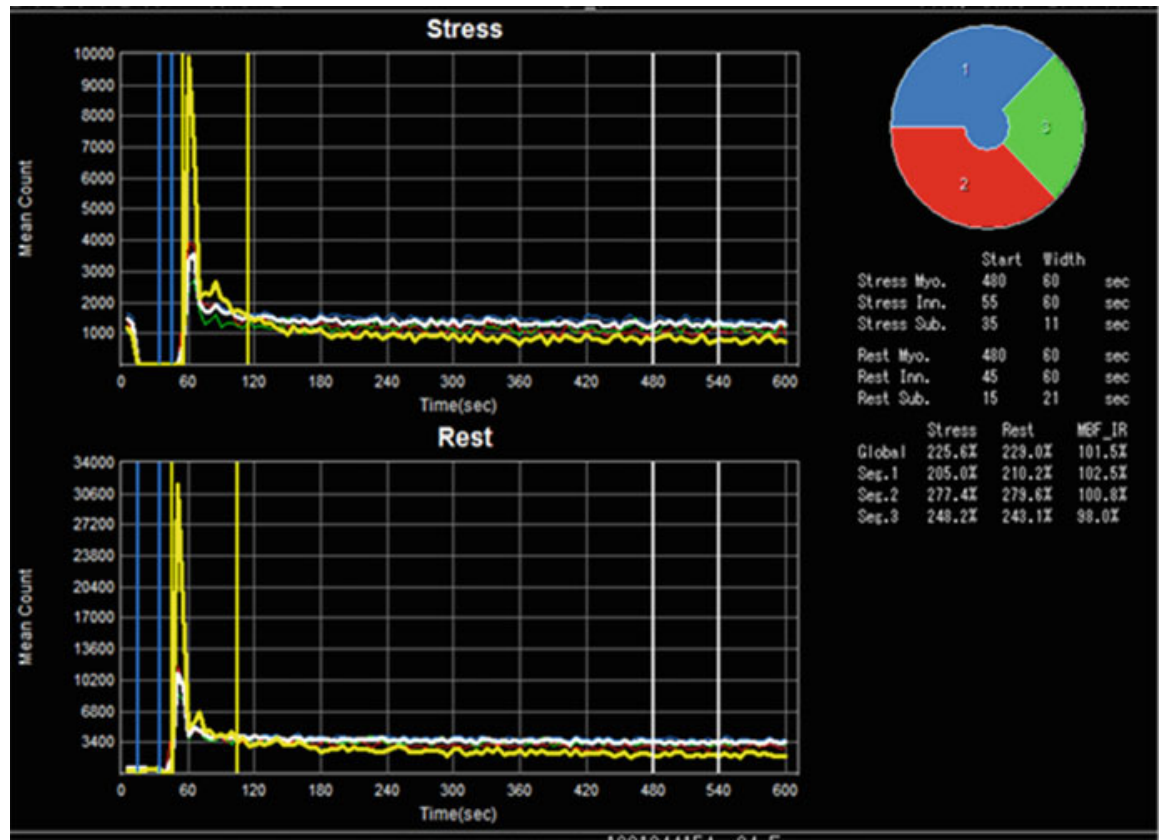

Fig. 14.3 Dynamic acquisition images were transferred to a workstation for analysis using a novel software. It allows an automatic definition of a volume of interest (VOI) for the blood pool in the left ventricle and the left ventricular myocardium. The time-activity curve (TAC) can be extracted by averaging the signal intensity in the VOI in each time frame and then expressed in counts per $\mathrm{mm}^{3} / \mathrm{s}$

with abnormal MPI. The CFR index was lower in territories supplied by stenotic coronary arteries than in non-stenotic arteries.

Novel software is currently under development in our institution [28]. TACs by dynamic ${ }^{99 \mathrm{~m}}$ Tc-tetrofosmin SPECT using the D530c during adenosine stress and rest are shown in Fig. 14.3. TAC can be extracted by averaging the signal intensity in the volume of interest (VOI) in each time frame and then expressed in counts per cubic $\mathrm{mm}^{3} / \mathrm{s}$. Global TAC was fitted to a 2-com model with input functions which was served by the blood pool curves. In the validation study, Global MFR index estimated by the 2-com model is significantly lower in patients with multi-vessel disease, than those without the disease. In addition, MFR index in the segments of vascular territories with significant coronary stenosis is significantly lower than those without coronary stenosis.

Most recently, Wells et al. applied the D530c to a study with a pig model for quantitation of absolute myocardial blood flow (MBF) using common perfusion tracers as ${ }^{99 \mathrm{~m}}$ Tc-MIBI, ${ }^{99 \mathrm{~m}}$ Tc-tetrofosmin, and thallium-201 [29]. Dynamic images were reconstructed with CT-based attenuation correction and energy window-based scatter correction and then processed with kinetic analysis using a 1-tissue 2-com model to obtain the uptake rate constant $\mathrm{K} 1$ as a function of microsphere MBF. Converting $\mathrm{K} 1$ back to $\mathrm{MBF}$ using the measured extraction fractions produced 
accurate values and good correlations with microsphere MBF. They have demonstrated that dynamic SPECT by the CZT camera may be feasible to estimate absolute MBF.

\subsubsection{Advantages and Disadvantages of the Measurement of CFR with SPECT}

At the current moment, PET plays a major role in accurate estimation of MBF or CFR. However, it has limited value for routine clinical studies because of its higher cost and more complicated procedures, including the production of short-half-lived positron tracers by in-house cyclotrons. Therefore, the advantage of SPECTmeasured technique utilizing common technetium-99 m perfusion tracers is that it would increase the utility of CFR measurement in the clinical setting with a much smaller financial cost.

On the other hand, the disadvantage of quantifying CFR with SPECT may be the underestimation of the CFR value, compared to that with PET. The reasons for this underestimation could be mainly due to the limited extraction of technetium- $99 \mathrm{~m}$ perfusion tracers at high flow rates, at which the extraction of the tracer becomes limited by membrane transport [26].

\subsection{Conclusions}

We need to explore patient-centered, radiation exposure-controlled, and appropriately designed protocols which do not sacrifice image quality or diagnostic accuracy of the new modality. Although ${ }^{99 \mathrm{~m}} \mathrm{Tc}$-labeled radiotracers might not be ideal flow agents, estimation of absolute MBF or CFR using dynamic CZT SPECT is challenging and of great interest. This technology will hold great promise if the several issues can be solved through future studies.

Open Access This chapter is distributed under the terms of the Creative Commons AttributionNoncommercial 2.5 License (http://creativecommons.org/licenses/by-nc/2.5/) which permits any noncommercial use, distribution, and reproduction in any medium, provided the original author(s) and source are credited.

The images or other third party material in this chapter are included in the work's Creative Commons license, unless indicated otherwise in the credit line; if such material is not included in the work's Creative Commons license and the respective action is not permitted by statutory regulation, users will need to obtain permission from the license holder to duplicate, adapt or reproduce the material. 


\section{References}

1. McNulty EJ, Hung Y, Almers LM, Go AS, Yeh RW. Population trends from 2000-2011 in nuclear myocardial perfusion imaging use. JAMA. 2014;311:1248-9.

2. National Council on Radiation Protection and Measurements. Report No. 160, Ionizing Radiation Exposure of the Population of the United States: National Council on Radiation Protection and Measurements; 2009.

3. Einstein AJ. Effects of radiation exposure from cardiac imaging: how good are the data? J Am Coll Cardiol. 2012;59:553-65.

4. Cerqueira MD, Allman KC, Ficaro EP, et al. Recommendations for reducing radiation exposure in myocardial perfusion imaging. J Nucl Cardiol. 2010;17:709-18.

5. DePuey EG, Mahmarian JJ, Miller TD, et al. Patient-centered imaging, ASNC Preferred Practice Statement. J Nucl Cardiol. 2012;19:185-215.

6. Bocher M, Blevis IM, Tsukerman L, Shrem Y, Kovalski G, Volokh L. A fast cardiac gamma camera with dynamic SPECT capabilities: design, system validation and future potential. Eur J Nucl Med Mol Imaging. 2010;37:1887-902.

7. Patton J, Slomka P, Germano G, Berman D. Recent technologic advances in nuclear cardiology. J Nucl Cardiol. 2007;14:501-13.

8. Takahashi Y, Miyagawa M, Nishiyama Y, Ishimura H, Mochizuki T. Performance of a semiconductor SPECT system: comparison with a conventional Anger-type SPECT instrument. Ann Nucl Med. 2013;27:11-6.

9. Takahashi Y, Miyagawa M, Nishiyama Y, Kawaguchi N, Ishimura H, Mochizuki T. Dual radioisotopes simultaneous SPECT of ${ }^{99 \mathrm{~m}}$ Tc-tetrofosmin and ${ }^{123} \mathrm{I}$-BMIPP using a semiconductor detector. Asia Oceania J Nucl Med Biol. 2014;3:43-9.

10. Nishiyama Y, Miyagawa M, Kawaguchi N, et al. Combined supine and prone myocardial perfusion single-photon emission computed tomography with a cadmium zinc telluride camera for detection of coronary artery disease. Circ J. 2014;78:1169-75.

11. Hindorf C, Oddstig J, Hedeer F, Hansson MJ, Jögi J, Engblom H. Importance of correct patient positioning in myocardial perfusion SPECT when using a CZT camera. J Nucl Cardiol. 2014;21:695-702.

12. Oddstig J, Hedeer F, Jogi J, Carlsson M, Hindorf C, Engblom H. Reduced administered activity, reduced acquisition time, and preserved image quality for the new CZT camera. $\mathrm{J}$ Nucl Cardiol. 2013;20:38-44.

13. Duvall WL, Wijetunga MN, Klein TM, et al. Stress-only Tc-99m myocardial perfusion imaging in an Emergency Department Chest Pain Unit. J Emerg Med. 2011;42:642-50.

14. Einstein AJ, Johnson LL, DeLuca AJ, et al. Radiation dose and prognosis of ultra-low-dose stress-first myocardial perfusion SPECT in patients with chest pain using a high-efficiency camera. J Nucl Med. 2015;56:545-51.

15. Imbert L, Poussier S, Franken PR, et al. Compared performance of high-sensitivity cameras dedicated to myocardial perfusion SPECT: a comprehensive analysis of phantom and human images. J Nucl Med. 2012;53:1897-903.

16. Yoshinaga $\mathrm{K}$, Chow BJ, Williams $\mathrm{K}$, et al. What is the prognostic value of myocardial perfusion imaging using rubidium-82 positron emission tomography? J Am Coll Cardiol. 2006;48:1029-39.

17. Herzog BA, Husmann L, Valenta I, et al. Long-term prognostic value of ${ }^{13} \mathrm{~N}$ ammonia myocardial perfusion positron emission tomography added value of coronary flow reserve. $\mathrm{J}$ Am Coll Cardiol. 2009;54:150-6.

18. Murthy VL, Naya M, Foster CR, et al. Improved cardiac risk assessment with noninvasive measures of coronary flow reserve. Circulation. 2011;124:2215-24.

19. Naya M, Murthy VL, Taqueti VR, et al. Preserved coronary flow reserve effectively excludes high-risk coronary artery disease on angiography. J Nucl Med. 2014;55:248-55. 
20. Taki J, Fujino S, Nakajima K, et al. Tc-99m retention characteristics during pharmacological hyperemia in human myocardium: comparison with coronary flow reserve measured by Doppler flowire. J Nucl Med. 2001;42:1457-63.

21. Sugihara H, Yonekura Y, Kataoka K, Fukai D, Kitamura N, Taniguchi Y. Estimation of coronary flow reserve with the use of dynamic planar and SPECT images of Tc- $99 \mathrm{~m}$ tetrofosmin. J Nucl Cardiol. 2001;8:575-9.

22. Ito $\mathrm{Y}$, Katoh C, Noriyasu K, et al. Estimation of myocardial blood flow and myocardial flow reserve by 99 mTc-sestamibi imaging: comparison with the results of $\mathrm{O}-15 \mathrm{H}_{2} \mathrm{O}$ PET. Eur J Nucl Med Mol Imaging. 2003;30:281-7.

23. Storto G, Cirillo P, Vicario ML, et al. Estimation of coronary flow reserve by Tc-99m sestamibi imaging in patients with coronary artery disease: comparison with the results of intracoronary Doppler technique. J Nucl Cardiol. 2004;11:682-8.

24. Daniele S, Nappi C, Acampa W, et al. Incremental prognostic value of coronary flow reserve assessed with single-photon emission computed tomography. J Nucl Cardiol. 2011;18:612-9.

25. Iida H, Eberl S, Kim KM, et al. Absolute quantitation of myocardial blood flow with ${ }^{201} \mathrm{Tl}$ and dynamic SPECT in canine: optimization and validation of kinetic modeling. Eur J Nucl Med Mol Imaging. 2008;35:896-905.

26. Petretta M, Soricelli A, Storto G, Cuocolo A. Assessment of coronary flow reserve using single photon emission computed tomography with technetium 99m-labeled tracers. J Nucl Cardiol. 2008;15:456-65.

27. Ben-Haim S, Murthy VL, Breault C, et al. Quantification of myocardial perfusion reserve using dynamic SPECT imaging in humans: a feasibility study. J Nucl Med. 2013;54:873-9.

28. Miyagawa M, Nishiyama Y, Kawaguchi N, et al. Estimation of myocardial flow reserve using a cadmium-zinc telluride (CZT) SPECT in patients with multi-vessel coronary artery disease. J Nucl Med 2013:54;Supplement 157P.

29. Wells RG, Timmins R, Klein R, et al. Dynamic SPECT measurement of absolute myocardial blood flow in a porcine model. J Nucl Med. 2014;55:1685-91. 